\title{
Design of a Microwave Assisted Discharge Inductive Plasma Accelerator
}

\author{
SP-2010-1842709 \\ Presented at Space Propulsion 2010, San Sebastián, Spain \\ 3-6 May, 2010 \\ Ashley K. Hallock* \\ Princeton University, Princeton, NJ 08544 USA \\ Kurt A. Polzin ${ }^{\dagger}$ \\ NASA - Marshall Space Flight Center, Huntsville, AL 35812 USA
}

\begin{abstract}
A new plasma accelerator concept that employs electrodeless plasma preionization and pulsed inductive acceleration is presented. Preionization is achieved through an electron cyclotron resonance discharge that produces a weakly-ionized plasma at the face of a conical theta pinch-shaped inductive coil. The presence of the preionized plasma allows for current sheet formation at lower discharge voltages than those found in other pulsed inductive accelerators. The location of an electron cyclotron resonance discharge can be controlled through the design of the applied magnetic field in the thruster. A finite-element model of the magnetic field was used as a design tool, allowing for the implementation of an arrangement of permanent magnets that yields a small volume of preionized propellant at the coil face. This allows for current sheet formation at the face of the inductive coil, minimizing the initial inductance of the pulse circuit and maximizing the potential efficiency of the new accelerator.
\end{abstract}

\section{NOMENCLATURE}

$\begin{array}{llll}\mathbf{B}, B, B_{r}, B_{z} & \text { Magnetic field }(\mathrm{T}) & m_{e} & \text { electron mass }(\mathrm{kg}) \\ c & \text { speed of light }(\mathrm{m} / \mathrm{s}) & n_{e} & \text { electron number density }\left(\mathrm{m}^{-3}\right) \\ e & \text { elementary charge }(\mathrm{C}) & \epsilon & \text { permittivity of free space }(\mathrm{F} / \mathrm{m}) \\ \mathbf{f}, f_{r}, f_{z} & \text { Lorentz body force density }\left(\mathrm{N} / \mathrm{m}^{2}\right) & \eta_{\mathrm{t}} & \text { thrust efficiency } \\ \mathbf{j}, j_{\theta} & \text { Current density }\left(\mathrm{A} / \mathrm{mm}^{2}\right) & \omega & \text { Applied EM frequency }(\mathrm{rad} / \mathrm{s}) \\ k & \text { wave vector }\left(\mathrm{m}^{-1}\right) & \omega_{c} & \text { electron cyclotron frequency }(\mathrm{rad} / \mathrm{s}) \\ \Delta L & \text { change in circuit inductance }(\mathrm{H}) & \omega_{p} & \text { plasma frequency }(\mathrm{rad} / \mathrm{s}) \\ L_{0} & \text { circuit or parasitic inductance }(\mathrm{H}) & \omega_{U H} & \text { upper hybrid frequency }(\mathrm{rad} / \mathrm{s})\end{array}$

\section{INTRODUCTION}

$\mathrm{T}$ is desirable to extend the lifetime and increase the reliability of the propulsion system as much as possible due to the fact that maintenance or replacement of that system becomes particularly challenging once it has been launched into orbit from the surface of the Earth. In addition, the amount of payload as a percentage of the total vehicle mass can be increased if the size and mass of a propulsion system, including the propellant required to complete a mission, can be reduced. The use of electric propulsion (EP) results in a reduction in the amount of propellant needed for a

\footnotetext{
${ }^{*}$ Graduate Student, Mechanical and Aerospace Engineering Department, ahallock@princeton.edu.

†Propulsion Research Engineer, Propulsion Research and Technology Applications Branch, Propulsion Systems Department, kurt.a.polzin@nasa.gov.
} 
given mission because of the high values of specific impulse associated with EP as compared to other conventional propulsion systems.

Pulsed inductive plasma thrusters[1-4] are spacecraft propulsion devices in which electrical energy is capacitively stored and then discharged through an inductive coil. The thruster is electrodeless, with a time-varying current in a coil interacting with a plasma covering the face of the coil to induce a plasma current. Propellant is accelerated and expelled at a high exhaust velocity $(\mathcal{O}(10 \mathrm{~km} / \mathrm{s}))$ by the Lorentz body force arising from the interaction of the magnetic field and the induced plasma current.

Thrusters of this type possess many demonstrated and potential benefits that make them worthy of continued investigation. The electrodeless nature of these thrusters eliminates the lifetime and contamination issues associated with electrode erosion in conventional electric thrusters. Also, a wider variety of propellants are available for use when compatibility with metallic electrodes in no longer an issue. Pulsed inductive accelerators have been successfully operated using propellants like ammonia, hydrazine, and $\mathrm{CO}_{2}$, and there is no fundamental reason why they would not operate on other propellants like $\mathrm{H}_{2} \mathrm{O}$. It is well-known that pulsed accelerators can maintain constant specific impulse $I_{\mathrm{sp}}$ and thrust efficiency $\eta_{\mathrm{t}}$ over a wide range of input power levels by adjusting the pulse rate to maintain a constant discharge energy per unit pulse. It has also been demonstrated that a pulsed inductive thruster can operate in a regime where $\eta_{\mathrm{t}}$ is relatively constant over a wide range of $I_{\mathrm{sp}}$ values. Finally, thrusters in this class have operated at high energy per pulse, and by increasing the pulse rate they offer the potential to process very high levels of power using a single thruster.

The capacitors in some inductive accelerators, like the Pulsed Inductive Thruster[1,2], must be charged to high voltages so that the discharge through the inductive coil can first ionize the propellant. One way to alleviate this high voltage requirement of is to partially ionize the propellant in front of the inductive coil such that the induced electric field only needs to perform an under-voltage breakdown of already partially-ionized propellant[5], allowing the energy storage system to be initially charged to a more reasonable voltage level. Not all of the propellant must be preionized because only the preionized propellant within a few characteristic length scales of the inductive coil has any significant interaction with induced fields. The use of preionization is ubiquitous throughout literature on pulsed inductive devices with a wide range of applications, including plasma fusion as well as space propulsion. For example, preionization has been successfully employed by striking a discharge between two appropriately placed electrodes $[6,7]$, sending a separate lower-energy pulse through an inductive coil before the main discharge [8,9], and creating a seed plasma in a planar pulsed inductive accelerator $[4,10]$.

In this work, we present a new pulsed inductive accelerator concept that employs an electron cyclotron resonance (ECR) discharge to preionize the propellant. The thruster, called the Microwave Assisted Discharge Inductive Plasma Accelerator (MAD-IPA), is shown in Fig. 1. A static magnetic field in the thruster is carefully designed so the ECRdriven discharge is located on the face of the inductive coil, only preionizing the propellant in the region where the coil can strongly interact with it. The rest of this paper describes the design of an ECR ionization system and its subsequent incorporation into a pulsed inductive plasma accelerator.
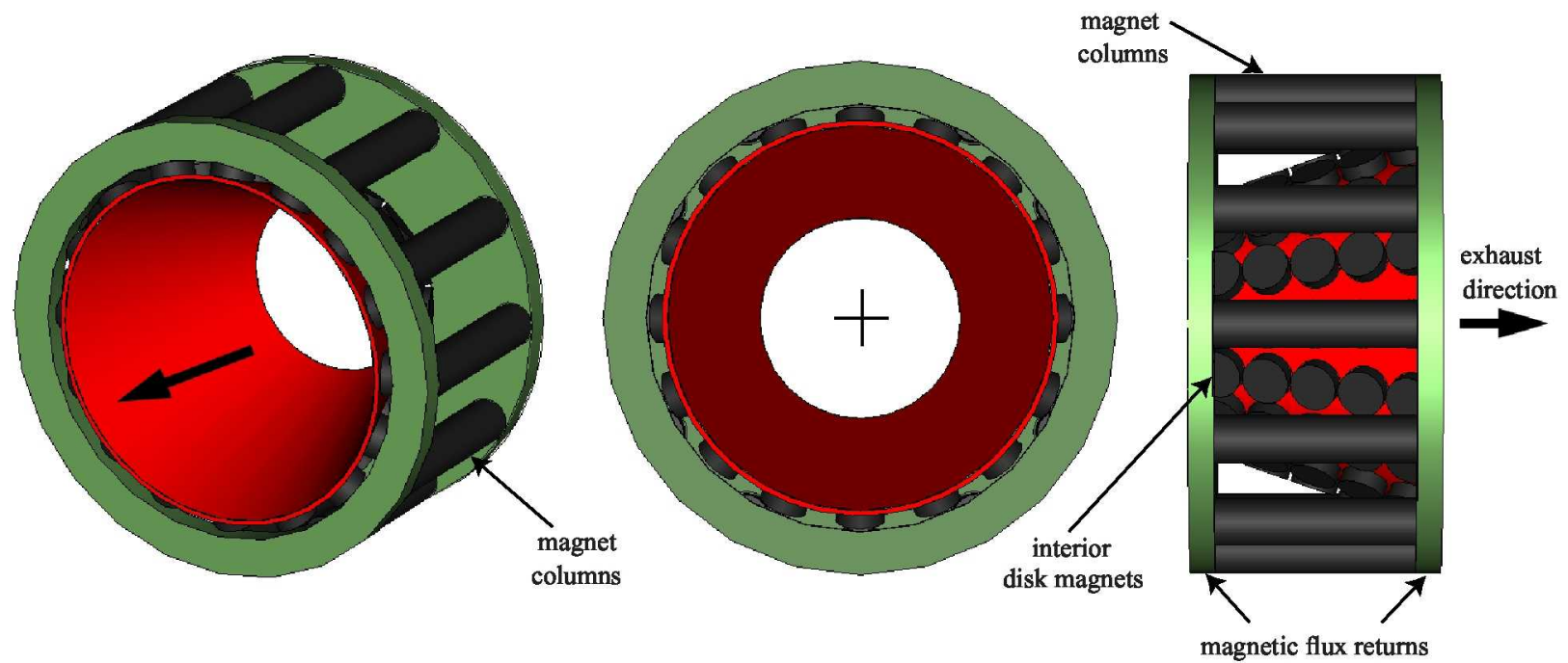

Figure 1. Multiple views of a finite element model of the MAD-IPA, with the inductive coil shown in red, the permanent magnets in gray, the ferrite pieces in green, and the thruster axis as a black arrow. 


\section{PREIONIZATION}

An ECR preionization scheme was chosen to produce a thin layer of plasma on the interior surface of a conicalshaped inductive coil because it offers the unique ability to control the location of ionization electrodelessly and without the imposition of high voltage-requirements, advantages of ECR that have previously been demonstrated in the Electrodeless Plasma Thruster [11-13]. The inductive coil over which the plasma forms has a minor radius of 4 $\mathrm{cm}$, a length of $10 \mathrm{~cm}$, and a cone half-angle of $20^{\circ}$. Plasma current formation only requires a thin layer of preionized plasma, just thick enough to allow for the formation of an inductively-coupled current sheet. As the current sheet forms and is inductively accelerated, the external circuit inductance will change from an initial value $L_{0}$ to a final value $L_{0}+\Delta L$. It is well known in the field of pulsed electromagnetic acceleration that the value of the ratio $\Delta L / L_{0}$ must be large in an efficient electromagnetic accelerator [14]. This ratio is maximized when the preionized plasma forms as close to the inductive coil as possible, but it should not form so closely that it causes insulator ablation. In the remainder of this section we describe the subsystems required to support an ECR discharge capable of satisfying these requirements.

\section{A. ECR Energy Addition Using Microwaves}

In the ECR process, energy is transferred from an electromagnetic (EM) wave to the electrons in the plasma. The wave energy can be focused into a given part of the plasma if a resonant condition can be established. The resonance condition can be determined by setting the wave number $k$ equal to zero in the dispersion relation for the wave and solving for the frequency $\omega$ of the wave. Of the four principal waves that propagate in a magnetized plasma, only the extraordinary wave ( $\mathrm{X}$ wave) and the right-hand polarized wave ( $\mathrm{R}$ wave) are capable of significant energy exchange with electrons in the plasma.

The $\mathrm{X}$ wave is defined as a wave where the wave vector is perpendicular to the applied magnetic field $B$ and the electric field is ellipically polarized relative to $B$. The $\mathrm{X}$ wave dispersion relation is

$$
\frac{c^{2} k^{2}}{\omega^{2}}=1-\frac{\omega_{p}^{2}}{\omega^{2}}\left[\frac{\omega^{2}-\omega_{p}^{2}}{\omega^{2}-\omega_{U H}^{2}}\right],
$$

where $c$ is the speed of light. The plasma frequency $\omega_{p}$ is defined as

$$
\omega_{p}=\frac{n_{e} e^{2}}{\epsilon_{0} m_{e}}
$$

where $n_{e}$ is the electron number density, $e$ is the charge on an electron, $\epsilon_{0}$ is the permittivity of free space, and $m_{e}$ is the mass of an electron. The upper hybrid frequency $\omega_{U H}$ is given as

$$
\omega_{U H}=\left(\omega_{p}^{2}+\omega_{c}^{2}\right)^{\frac{1}{2}}
$$

where $\omega_{c}$ is the electron cyclotron frequency:

$$
\omega_{c}=\frac{e B}{m_{e}}
$$

Resonance for the $\mathrm{X}$ wave occurs at $\omega=\omega_{U H}$, a condition which causes the right hand side of Eq. 1 to equal infinity.

The $\mathrm{R}$ wave is defined as having $k \| B$ with an electric field component that is perpendicular to $B$ and rotates around $B$ in the same sense as the electrons in the plasma. The dispersion relation for $\mathrm{R}$ waves is:

$$
\frac{c^{2} k^{2}}{\omega^{2}}=1-\frac{\omega_{p}^{2} / \omega^{2}}{1-\omega_{c} / \omega}
$$

Resonance for this wave occurs when $\omega=\omega_{c}$ which, unlike the $\mathrm{X}$ wave resonance, depends only upon the value of $B$. It is this resonance that we will take advantage of in the MAD-IPA, controlling the preionized plasma location solely through careful design of the background magnetic field profile. It should be noted that regardless of the polarization of the EM waves launched into the plasma, $R$ waves will form and propagate in the plasma in response to any launched waves whose wave vector is mostly aligned with the background magnetic field. In general, the restriction of wave emission to a single polarization has been shown to provide no benefit [15]. In our ECR scheme, this should hold true due to the fact that any waves in the plasma that do not propagate as $\mathrm{R}$ waves have a certain probability of being 
transformed into $\mathrm{R}$ waves through reflections with one of the metallic surfaces of the inductive coil. Therefore, no attempt was made in the design of the EM wave delivery system to control the polarization of the waves.

In the present MAD-IPA experiment, waveguides carry microwave energy from a magnetron source through a waveguide pressure window to a wave-launcher that feeds microwaves to the interior volume contained by the conical inductive coil. A rendering and photograph of the waveguide system on the inside of the vacuum vessel are shown in Fig. 2. It is known that coaxial microwave cables can arc at power levels as low as 100 Watts [13], so we instead chose to employ waveguides, allowing for testing over a wide range of input power levels. While only the TE 10 mode propagates through the rectangular waveguide sections, once the wave transitions to the cylindrical, coaxial wavelauncher, all field polarization is lost. The waves are emitted with a random orientation from the end of the launcher into the volume enclosed by the inductive coil.
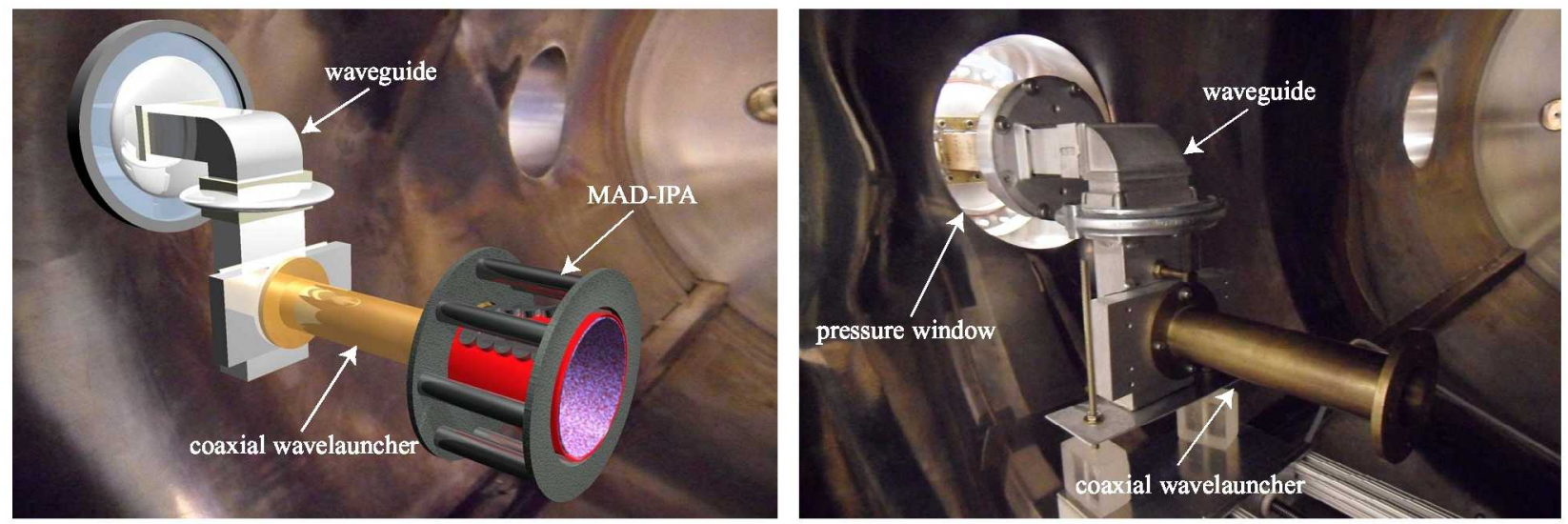

Figure 2. Left: Rendering of the waveguide setup connected to the thruster inside the vacuum vessel. Right: Photograph of the waveguide setup inside the vacuum vessel.

In future engineering and flight units, the magnetron and waveguide system could be replaced by surface-mount microwave resonator circuits (not unlike those commonly found inside cell phones) placed along the inside of the inductive coil. The signal from these circuits could be amplified if needed to provide a level of preionization sufficient to allow inductive current sheet formation for a particular pulse energy and capacitor bank charging voltage. Another advantage to this design is that the EM waves travel radially towards the thruster axis across a background magnetic field that decreases in magnitude at smaller radii, preventing wave reflections that might have otherwise occurred before the wave reached the location for resonance interaction. The only disadvantage to this design would be the addition of microwave circuits (and associated insulation) between the inductive coil traces and the plasma, slightly increasing the distance between the initial current sheet and the inductive coil, leading to an increased $L_{0}$.

\section{B. Applied Magnetic Field for ECR}

The applied magnetic field can be produced using electromagnets, permanent magnets, or a combination of the two. Permanent magnets were chosen due to the additional weight and power penalties associated with the use of electromagnets.

The resonance value for the applied magnetic field can be found by equating the electron cyclotron frequency, defined in Eq. 4, to the frequency of the EM waves. For an EM wave with a frequency of $2.45 \mathrm{GHz}$, the resonant magnetic field value is about $875 \mathrm{G}$. Therefore the applied magnetic field profile should be designed such that the absolute value of the field is equal to this value only in the space directly in front of the inductive coil, with a rapid decrease in $B$ in the direction normal to the surface of the coil toward the interior of the thruster. The resonance zone should also not intersect the inductive coil to help keep the plasma from interacting with and ablating the insulating surface in front of the inductive coil. In locations where the magnetic field does bend towards or intersect the inductive coil, the field strength should be great enough to reflect, or magnetically mirror, the preionized plasma away from the surface.

The direction of the magnetic field should also parallel that of the self magnetic field created by the plasma current, increasing the Lorentz body force density $f$ that accelerates the propellant, which is related to both the plasma current density $\mathbf{j}$ and the magnetic field $\mathbf{B}$ : 


$$
\mathbf{f}=\mathbf{j} \times \mathbf{B} \text {. }
$$

This equation can be separated into an axial and a radial component:

$$
f_{z}=j_{\theta} B_{r} \quad \text { and } \quad f_{r}=j_{\theta} B_{z} .
$$

With a properly contoured magnetic field profile, the permanent magnet assembly not only creates an ECR zone but also contributes to the axial and radial acceleration of plasma. However, if the magnetic field intersects the inductive coil at any point the field may partially cancel the induced magnetic field at that location, reducing the achievable thrust. In addition, if there is not enough magnetic mirroring to repel the plasma from the coil surface, the insulator may be ablated by the preionized propellant. Therefore, it is desirable that the magnetic field lines be approximately parallel to the inside surface of the inductive coil.

The desired magnetic field is produced in the following manner. First, axially-oriented columns of magnets bounded at their ends by ferrite discs create a fairly uniform magnetic field profile within the volume of the inductive coil that follows the geometry of the cone with minimal intersection points. This field has a value that is roughly half of the resonant value, and due to its geometry, may contribute to thrust. Next, disc magnets are laid flat in rows on the outer surface of the inductive coil to create a magnetic field that has a large gradient where the magnitude quickly decreases moving radially inward towards the thruster axis. While this field is mostly azimuthal, and will not contribute to thrust, the superposition of this field with that of the column magnets creates a profile where the value of the magnetic field close to the inductive coil is at resonance and then rapidly decreases in the direction normal to the inductive coil, reaching about half the resonant value only a few $\mathrm{mm}$ away from the inductive coil towards the thruster axis. This permanent magnet assembly is shown in Fig. 3 with finite element simulation results of the associated magnetic field profile.
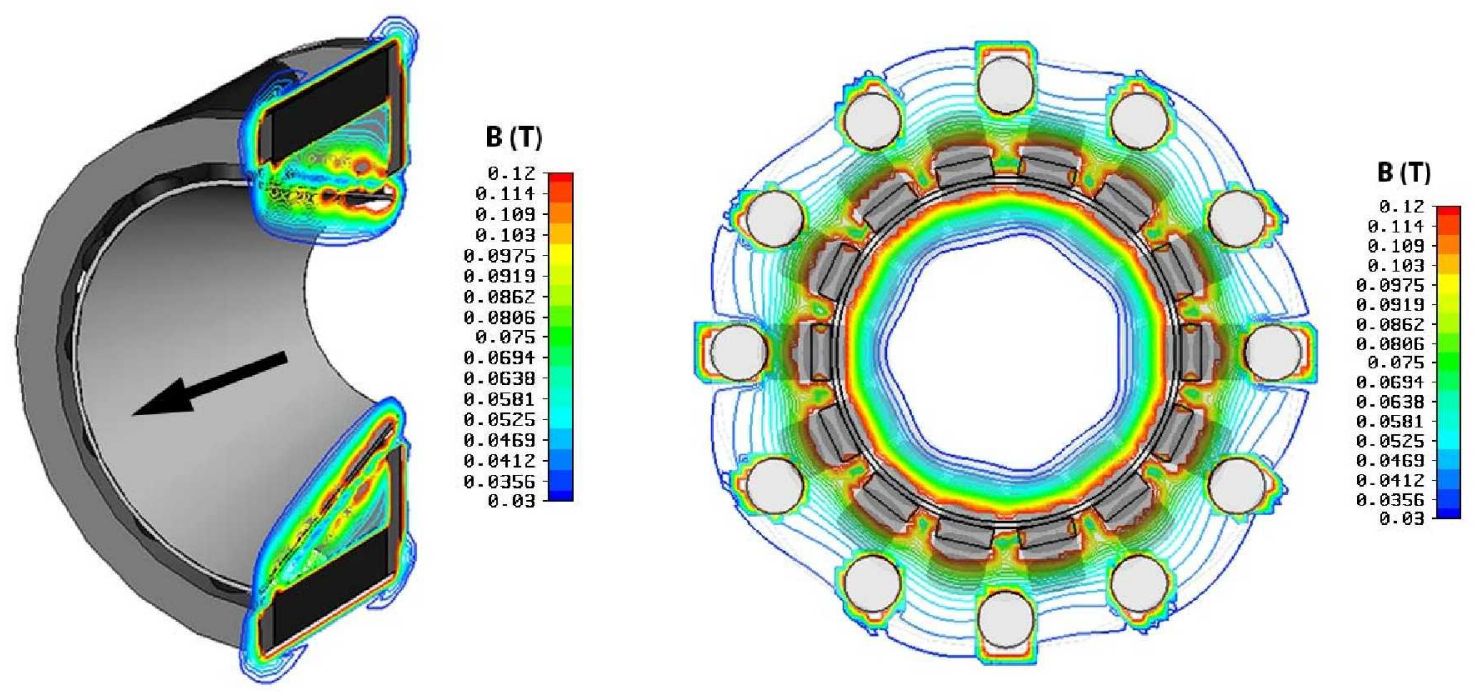

Figure 3. Finite element model of the magnetic field surrounding the MAD-IPA, with the resonance zone shown in yellow, green and light blue, and the thruster axis as a black arrow. The structure is shown in black and white to clarify the magnetic field contour. Left: Perspective view. Right: Front view along thruster axis.

\section{INDUCTIVE COIL}

The inductive coil, shown in Fig. 4 is in the shape of a conical theta pinch and is composed of spiral copper traces 0.2 $\mathrm{mm}$ thick that cover both sides of a $0.45 \mathrm{~mm}$ thick mylar conical frustum. The frustum has a minor radius of $4 \mathrm{~cm}, \mathrm{a}$ length of $10 \mathrm{~cm}$, and a half cone angle of $20^{\circ}$. An additional layer of mylar is added to the inner surface of the frustum to insulate the inner copper traces from the plasma. The pattern of the copper traces, shown in Fig. 5, is such that the radial and axial components of current from the two sides of the mylar will cancel, yielding a uniform, azimuthal current across the coil face (in a manner similar to that used in the Pulsed Inductive Thruster [1]). Current is fed to each trace individually via coaxial cables connected at the upstream end, or smaller end, of the frustum. The current 
follows an Archimedes spiral, completing two full turns while travelling along the entire axial length of the frustum twice before the path returns to the point of origin on the opposite side of the mylar insulation.
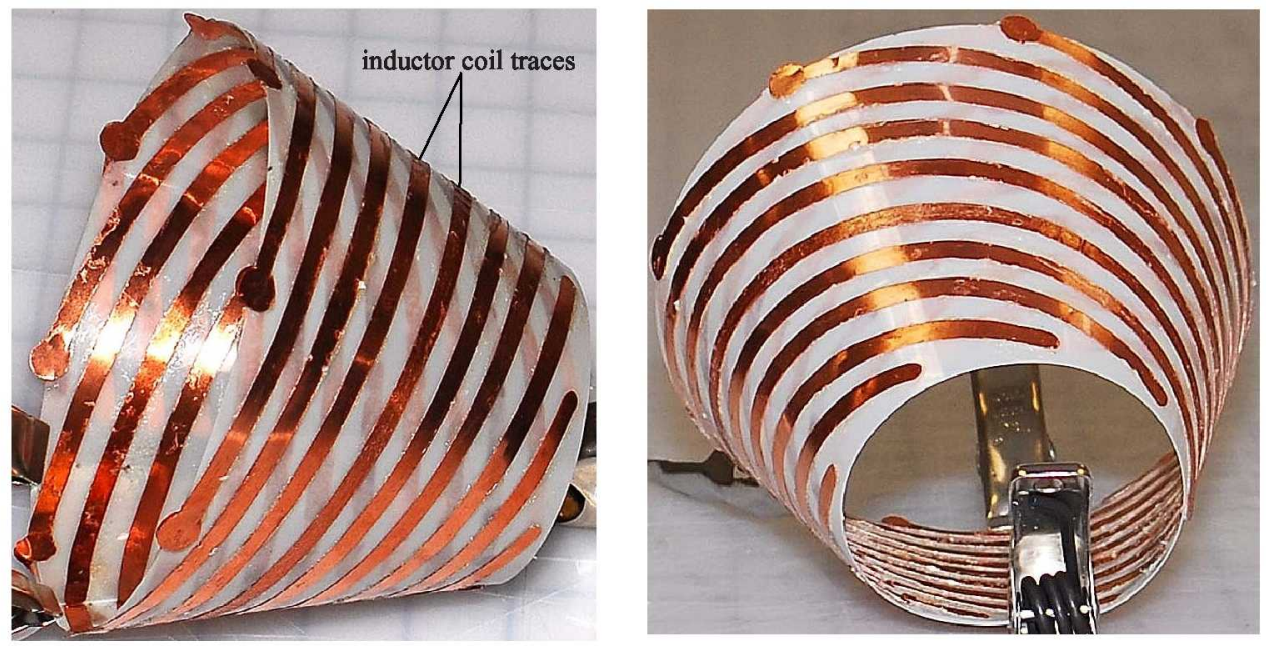

Figure 4. The inductive coil composed of copper traces shown adhered to a mylar sheet that provides insulation and structural support (light squares visible in the background of the left image measure one-inch square).
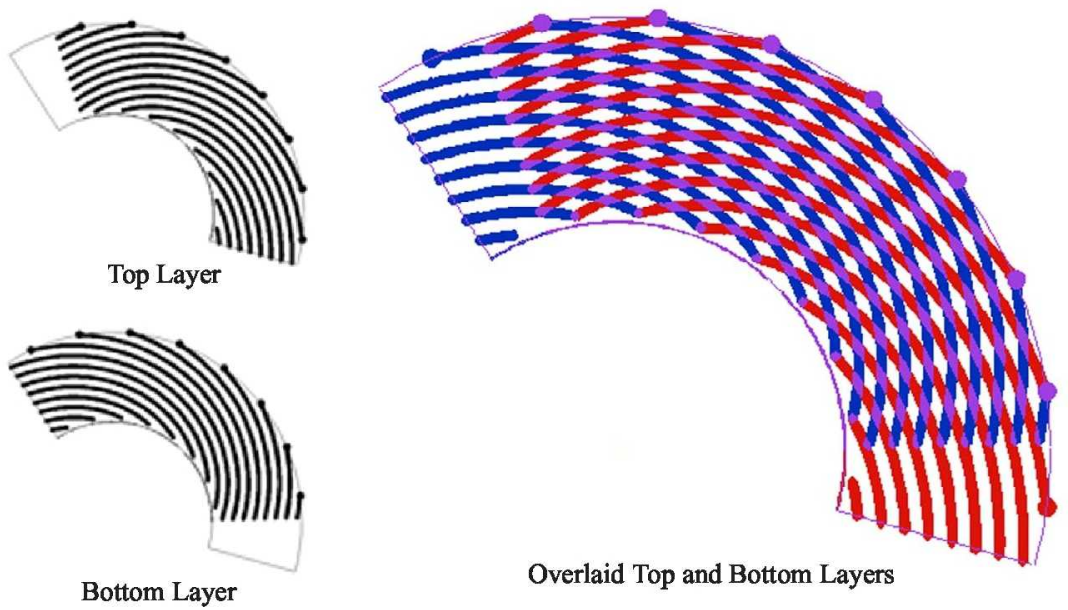

Figure 5. Copper trace design used to create the conical inductive coil. In the overlaid image, the top layer is shown in red, the bottom layer in blue, and where they overlap the trace is purple.

\section{CONCLUSION}

In this paper we presented a new plasma accelerator concept, the MAD-IPA, that uses an electrodeless ECR preionization scheme to produce a seed plasma that is further ionized and accelerated by an external current pulsed through an inductive coil. The current rise rate (inductive voltage drop) needed across the inductive coil during a pulse is lower when the field produced by the inductive coil is not required to ionize a neutral gas. This allows for a lowering of the charge voltage in the MAD-IPA relative to other pulsed inductive concepts like the PIT. ECR was chosen in the interest of controlling the location and geometry of the propellant ionization region. Through finite element analysis, it was determined that an arrangement of commercially-available permanent magnets could be employed to produce a magnetic field topology that satisfies the design requirements of the MAD-IPA. This arrangement of permanent magnets yields a region along the inner surface of the coil that is favorable for an ECR discharge, and through an interaction between the induced plasma current and the applied magnetic field, additional thrust may also be realized. 


\section{ACKNOWLEDGMENTS}

The authors would like to thank Mr. Gregory Emsellem for aiding in the design of the magnetic field. We appreciate the help and support of Dr. Bill Emrich, Mr. J. Boise Pearson, and Mr. Jim Martin, and thank Mr. Tommy Reid, Mr. Douglas Galloway, and Mr. Adam Kimberlin for their invaluable technical assistance. This work was supported, in part, by U.S. Department of Energy Contract No. DE-AC02-76-CHO-3073.

\section{REFERENCES}

${ }^{1}$ Dailey, C. L. and Lovberg, R. H. The PIT MkV Pulsed Inductive Thruster. Technical Report 191155, Lewis Research Center, Redondo Beach, CA, July 1993.

${ }^{2}$ Lovberg, R. H. and Dailey, C. L. A PIT primer. Technical Report 005, RLD Associates, Encino, CA, 1994.

${ }^{3}$ Polzin, K. A. Faraday Accelerator with Radio-frequency Assisted Discharge (FARAD). Ph.D. dissertation, Princeton University, Department of Mechanical and Aerospace Engineering, 2006.

${ }^{4}$ Choueiri, E. Y. and Polzin, K. A. Faraday Acceleration with Radio-frequency Assisted Discharge. Journal of Propulsion and Power, 22(3):611-619, May-June 2006.

${ }^{5}$ Polzin, K. A. Scaling and System Considerations in Pulsed Inductive Plasma Thrusters. IEEE Transactions on Plasma Science, 36(5):21892198, October 2008.

${ }^{6}$ Josephson, V. and Hales, R. W. Parametric Study of the conical Shock Tube. The Physics of Fluids, 4(3):373-379, 1961.

${ }^{7}$ Cassibry, J. T. Fimognary, P. J. and Ims, K. E. Effects of Pre-ionization and Bias Field on Plasmoid Formation and Acceleration. Number AIAA-2007-5262, July 2007.

${ }^{8}$ Josephson, V. Production of High Velocity Shocks. Journal of Applied Physics, 29(1):30-32, 1958.

${ }^{9}$ Cruddace, R. C. and Hill, M. Mechanism of Plasma Acceleration in a Conical Theta-pinch Gun. Technical Report CLM-M52, Culham Laboratory, 1966.

${ }^{10}$ Miller, R. Best, S Owens, T. Polzin, K. A., Rose, F. M. and Dankanich, J. Design of a Low-Energy FARAD Thruster. Number AIAA 2007-5257, July 2007.

${ }^{11}$ Emsellem, G. D. and Larigaldie, S. Development of the Electrodeless Plasma Thruster at High Power: Investigation of the MicrowavePlasma Coupling. Number IEPC-2007-240, September 2007.

${ }^{12}$ Emsellem, G. D. and Larigaldie, S. Low Power Behavior of the Electrodeless Plasma Thruster. Number AIAA-2008-5009, July 2008.

${ }^{13}$ Emsellem, G. D. Coupling and Erosion Evaluation of the High Power Electrodeless Plasma Thruster at Low Power at Princeton University. Number AIAA-2009-5449, August 2009.

${ }^{14}$ Gooding, T. Lovberg, R. H., Hayworth, B. R. The Use of a Coaxial Gun for Plasma Propulsion. Technical Report AE62-0678, G. D. Convair, 1962.

${ }^{15}$ Geller, R. Electron Cyclotron Resonance Ion Sources and ECR Plasmas. Institute of Physics Publishing, 1996. 